Article

\title{
Communicating the Risks and Benefits of Human Urine-Derived Fertilizer
}

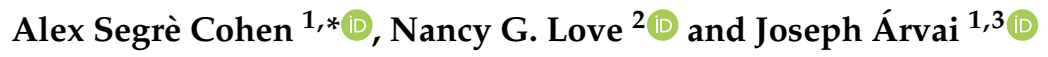 \\ 1 Department of Psychology, University of Southern California, Los Angeles, CA 90089, USA; arvai@usc.edu \\ 2 Department of Civil and Environmental Engineering, University of Michigan, Ann Arbor, MI 48103, USA; \\ nglove@umich.edu \\ 3 Decision Research, Eugene, OR 97401, USA \\ * Correspondence: acohen06@usc.edu
}

Received: 26 October 2020; Accepted: 24 November 2020; Published: 28 November 2020

check for updates

\begin{abstract}
Ensuring long-term access to nutrients needed for food production is a growing global challenge. Human urine diversion and recycling is a viable and energy-efficient means of recovering nitrogen, phosphorus, and potassium from wastewater. Before implementation, however, it is critical to understand how communicating differently about human urine-derived fertilizer may influence its public acceptance. This study tests how different strategies of communication (video compared to texts), as well as different amounts of information, impact public acceptance. We also explored how specific characteristics, such as age and education level, may impact the usefulness of the different strategies of communication. The results indicate that short and long videos are the most useful risk communication strategies, and age fully moderates this relationship. This research may serve as a jumping off point for future studies focused on how risk communication strategies may affect consumer acceptance of other emerging food technologies.
\end{abstract}

Keywords: risk communication; emerging technology; public acceptance; urine-diversion and reuse

\section{Introduction}

One of the most pressing natural resource challenges facing the world today is ensuring long-term access to nitrogen and phosphorus [1]. These nutrients are vital ingredients in fertilizers that have become essential in global agricultural systems [2,3]. Access to nitrogen and phosphorus is a growing concern, in part due to phosphate rock being a non-renewable resource [4] and in part because humans' use of nitrogen and phosphorus is inefficient [5]. Due to the severity and timeliness of these challenges, engineered solutions for recovering and recycling nitrogen and phosphorus from wastewater are emerging.

One of these solutions, human urine diversion and recycling, is a viable and energy-efficient means of recovering nitrogen and phosphorus (and potassium) from wastewater; the added benefit of this approach is that it yields nutrients for manufacturing agricultural fertilizers that mirror conventional options [6]. Diverting and recycling human urine to use as an agricultural fertilizer increases the sustainability of food production $[7,8]$ while also protecting waterways from nutrients and micropollutants which can be more easily removed from the concentrated urine than after it is diluted into wastewater. These human urine-derived fertilizers (HUDF) can be tailored to match the soil types and agronomy practices of sizable swatches of the world [9].

The technology required for diverting, recycling, and transforming human urine into fertilizer has been developed and is currently being implemented in a series of small-scale pilot initiatives [10-14]. However, the deployment of infrastructure for urine diversion and HUDF production at scale will require retrofitting or replacing both public and building-size wastewater systems. While technically 
feasible, retrofitting and rebuilding these systems are unlikely without broad public acceptance of HUDF use.

A relatively small number of studies on public acceptance of HUDF point to relatively high levels of receptivity to HUDF among users of the fertilizers themselves (e.g., farmers and other growers) and among users of the infrastructure required to collect human urine (e.g., people willing to use modified toilets). The majority of these studies have relied on qualitative methods, have been based on small samples of consumers and users, and have focused on HUDF deployment in Europe and Asia [15-18].

However, a recent survey conducted in the United States with a large, representative sample of American consumers [19] supports the assertion that public receptivity to HUDF may indeed be high. This research highlighted broad consumer openness to HUDF, especially when it was compared with other common fertilizers. However, in this study, consumer support for HUDF did vary by end use; specifically, receptivity to HUDF was highest for crops that are not intended for human consumption [19]. These findings suggest obstacles to the more widespread deployment of nutrient recapture and recycling via human urine diversion. Specifically, as consumers hear more-but do not necessarily learn more-about the technology, they may sour on the acceptability of HUDF for use on crops for human (and perhaps even non-human) consumption.

This phenomenon is not new in the domain of emerging technologies. Rapid and precipitous declines in public acceptability ratings have been observed for a wide range of emerging technologies. These declines are usually accompanied by growing public awareness of these technologies alongside social risk amplification [20,21]. Examples include nanotechnology [22], carbon capture and storage [23], atmospheric geoengineering [24], wastewater recycling [25], nuclear power [26], and nuclear waste disposal [27], among others. For each of these technologies, researchers and practitioners have argued that better risk communication could have helped to avoid the controversies about them that ensued.

The overreaching goal of risk communication for emerging technologies is to facilitate an understanding of their underlying processes, benefits, and risks (including how they are assessed and may be managed) so that people can make more informed and defensible judgments and decisions about them [28,29]. When done well, risk communication addresses cognitive gaps by correcting misunderstandings [30]. Successful risk communication-in that it is informative and supports judgment and decision-making-depends, however, on whether it addresses consumers' information needs by being both content-specific and also accessible.

In terms of content, risk communication should present relevant risks, benefits, and uncertainties clearly [30]. The information must also be accurate and thorough, such that it increases understanding. Most importantly, for risk communication to be effective, it must be responsive to the needs of the user [28]. This is not to say effective risk communication messages are overloaded with technical details; indeed, information-heavy risk communication does not necessarily lead to more informed participants or better decision-making [29]. Rather, designers and practitioners of risk communication must be attentive to essential gaps in understanding about risks, and must design content that both fills these gaps and-importantly-reinforces existing knowledge [29,31,32]. At the same time, risk communication that accounts for the established communication norms of its participants, alongside creative messaging, is more likely to capture people's attention, thereby increasing the likelihood that the information conveyed is both understood and put to use [29]. In this decade, for example, risk communication efforts that take advantage of social (e.g., Facebook, Twitter, YouTube, etc.) and digital (e.g., videos, podcasts, etc.) media are seen by many as more accessible than printed materials [33,34].

When designing risk communications, it is equally important to note that people from different generations and life stages, as well as those with different educational levels, typically do not process information in the same way. Thus, risk communication efforts around a single topic may require different risk messaging approaches such that they are carefully tailored to the needs of different groups. For example, young children and elderly adults are uniquely sensitive to the quantity of information provided, and may benefit from more straightforward and succinct materials [35]. 
In addition, how information is disseminated may affect the degree to which information in risk communication is internalized. For example, in medicine, patients who watch videos (vs. reading text) about treatment options have been shown to be more aware of both the risks and benefits associated with them [36]. Similarly, videos-in contrast to printed materials-have increased individual's understanding of the risks from climate change [37]. Other work also shows that risk messages that include either videos or photos influence risk perceptions more strongly than risk messages that rely on text alone [38]. Thus, different risk communication strategies (e.g., presenting information via video, text, or in-person) as well as individual's demographic factors may have moderating effects on the usefulness (e.g., by improving recipients' understanding of the risks, or leading to acceptance) of risk communication about HUDF.

In addition to the risk communication strategy employed, and the end use of HUDF, receptivity to it by consumers and other stakeholders may also depend upon a host of psychological considerations. For example, we know from prior research that people have a tendency to prefer natural options (e.g., when considering consumer products, or when evaluating such things as manufacturing processes and disturbances) over artificial or anthropogenic ones $[39,40]$. This preference can be traced to humans' inherent attachment to nature. This partiality can be so strong, in fact, that it creates a bias wherein people may opt for what is framed as "natural" over otherwise identical artificial engineered options [39].

We also know that intuitive, fast-acting emotions play a role in our perceptions, judgements, and ultimately our decisions [41,42]. One of these emotions, disgust, is especially relevant to research on food and food-related products. Disgust has an instinctive and rapid response linked to evolutionary origins. It historically served as a mechanism to keep animals from ingesting harmful items [43]. We know from both research $[43,44]$ and personal experience that human waste is typically disgust inducing. However, it remains to be seen if nutrients extracted, and fertilizers manufactured from human urine would be viewed as disgust inducing and if this, in turn, would negatively affect both risk communication efforts and consumers' receptivity to HUDF.

Finally, values serve as guides for judgments and decisions about emerging technologies such as nuclear power [45], nanotechnology [22], and solar radiation management [24]. Specifically, different value orientations (e.g., altruism, biospherism, and egoism) have been shown to lead to different degrees of receptivity to pro-environmental behavior and technologies [46]. For example, those who ascribe strongly with altruistic (i.e., a high level of concern for others) and biospheric (i.e., a high level of concern for the natural environment) values compared to those with egoistic (i.e., a high level of concern for one's personal well-being) value orientations tend to exhibit more pro-environmental behaviors and tend to be more accepting of technologies and policies with high environmental benefits [23].

With this as the backdrop, the objective of this research was to study the influence of alternative risk communication approaches on peoples' ratings of the usefulness of the information contained in them, and on their acceptance of HUDF for use in food production. Specifically, this study focused on how watching a risk communication video vs. reading a text-based presentation containing the same information may influence consumers' acceptance of HUDF. We were also interested in the effect of the length of these videos and texts and how it would influence consumers' acceptance of HUDF. In addition, we studied the potential moderating effects of age, education, and how individual characteristics, such as food disgust sensitivity and value orientations, impact the usefulness of the risk communication messages.

\section{Materials and Methods}

\subsection{Participants}

Participants were residents of the United States over the age of 18 recruited from a quasi-representative internet panel sourced by Qualtrics. Data collection took place in 2019. 
Quota sampling was applied to balance gender, age, and to randomly assign participants into one of five conditions (see Section 2.2 below). After consenting to participate, 4103 participants responded to the instrument. A total of 753 participants were removed by Qualtrics because they did not complete the study $(n=743)$ or because they began the survey after quotas were achieved $(n=10)$. An additional 873 participants were removed because they failed a series of instructed choice attention checks $(n=400)$; because they spent less than half the median time (00:04:01) on the instrument $(n=264)$; or because they selected the same response on all items of a 13-item scale and/or an 8-item scale $(n=209)$. The final sample size was 2477 , which provided adequate statistical power [47].

Across the five risk communication strategies, $50 \%$ of respondents were women; the mean age of participants was 40-48 years and the median education level corresponded with self-reports of "associate degree" (Table 1). These demographic characteristics are emblematic of an "average" American [48].

Table 1. Sample characteristics. Individual education levels were combined and categorized as low (no high school + some high school + graduated high school/GED), medium (some college + associate degree), or high (bachelor's degree + graduate/professional degree).

\begin{tabular}{|c|c|c|c|c|c|c|c|c|c|c|}
\hline & $n$ & Women & $\begin{array}{c}\text { Age } \\
(18-29)\end{array}$ & $\begin{array}{c}\text { Age } \\
(30-39)\end{array}$ & $\begin{array}{c}\text { Age } \\
(40-48)\end{array}$ & $\begin{array}{c}\text { Age } \\
(49-59)\end{array}$ & $\begin{array}{c}\text { Age } \\
(60+)\end{array}$ & $\begin{array}{c}\text { Low } \\
\text { Education }\end{array}$ & $\begin{array}{l}\text { Medium } \\
\text { Education }\end{array}$ & $\begin{array}{c}\text { High } \\
\text { Education }\end{array}$ \\
\hline Control & 510 & $52 \%$ & $18 \%$ & $18 \%$ & $20 \%$ & $22 \%$ & $22 \%$ & $18 \%$ & $30 \%$ & $52 \%$ \\
\hline Short text & 515 & $49 \%$ & $18 \%$ & $19 \%$ & $20 \%$ & $21 \%$ & $21 \%$ & $20 \%$ & $33 \%$ & $47 \%$ \\
\hline Long video & 506 & $50 \%$ & $17 \%$ & $20 \%$ & $21 \%$ & $21 \%$ & $22 \%$ & $17 \%$ & $33 \%$ & $50 \%$ \\
\hline Long text & 443 & $49 \%$ & $20 \%$ & $19 \%$ & $19 \%$ & $20 \%$ & $21 \%$ & $20 \%$ & $32 \%$ & $50 \%$ \\
\hline
\end{tabular}

\subsection{Design}

To address our research objectives, participants first provided their informed consent and then were randomly assigned to one of five different risk communication treatment groups: (1) a control comprised of a brief, two-sentence description of agricultural fertilizers and HUDF; (2) a 6.5 min animated video; (3) a short version (3.7 min) of the video used in treatment 2; (4) information in text form based on the complete script for the long video used in treatment 2; (5) information in text form based on the complete script for the short video used in treatment 3 . The treatments vary in terms of how detailed and in which format the information is given but they have been constructed to all contain the same content. Each of the four risk communication strategies (apart from the control) provided detailed information about why fertilizers are used in agriculture, technological advancements leading to human urine diversion and nutrient recycling, HUDF production, HUDF as an alternative to conventional fertilizers, and information about the benefits, risks, and uncertainties associated with HUDF use. The information in the videos used in strategies 2 and 3 was conveyed by an animated narrator ("Uri") who was portrayed as anthropomorphized drop of human urine.

The choice to use an animated video featuring Uri as the narrator was made before this research was designed and conducted. Though the video was designed with best practices in risk communication in mind, it was produced to inform external stakeholders and partners about the broader National Science Foundation-funded initiative (under the Innovations at the Nexus of Food, Energy and Water Systems program) to study nutrient recycling and HUDF. It was later, when the first author joined the project, that we decided to use the video as the basis for a broader study on risk communication and consumer acceptance of HUDF. The videos and related texts were developed by Linda Macpherson and reviewed for content and accuracy by a group of geoscientists, ecologists, and agronomists from a multidisciplinary study team associated with other elements of this HUDF research initiative. This study was approved by the Health Sciences and Behavioral Sciences Institutional Review Board (protocol number HUM00156157) at the University of Michigan. 
At the start of the instrument, the risk communication treatments were immediately followed by an attention check to determine whether respondents could correctly identify the fertilizer type they just learned about. The same survey instrument was then shown to each participant, regardless of their treatment group. Each strategy (including links to the videos) and the survey questions can be found in the Supplemental Materials.

To study the effects of the risk communication treatments on participants' acceptance of HUDF, we posed questions for four distinct end-uses. Specifically, we asked for participant's level of agreement with the statement "It is acceptable to use urine-derived fertilizer to grow [non-edible plants like flowers]"; the remaining three questions asked about "fruits and vegetables that will be eaten by only animals"; "plants, trees, and grasses that grow around my home"; "fruits and vegetables for people to eat". Responses for all four questions were collected for each fertilizer type on 7-point Likert scales from "strongly disagree" to "strongly agree" (midpoint = "neither disagree nor agree"). The three statements about HUDF use on agricultural products that are not intended for human consumption were combined to create an index (Cronbach's $\alpha=0.85$ ).

Next, we posed a series of questions focused on how useful participants found the different risk communication formats to be. Specifically, participants were asked to respond to the following statements on 7-point Likert scales ranging from "Not at all" to "Extremely" (the midpoint was not labeled): (1) "I found the information about urine-derived fertilizer to be interesting"; (2) "I found the information about urine-derived fertilizer to be informative"; (3) "I found the information about urine-derived fertilizer to be frustrating"; (4) "I found the information about urine-derived fertilizer to be worrisome." Responses to questions 3 and 4 were reverse coded; all responses were combined to create an index of "usefulness" (Cronbach's $\alpha=0.77$ ).

The effect of the risk communication treatments on perceived risks and benefits of HUDF was measured using four questions; two questions concerned risks and benefits to environmental health, and two questions concerned human health. Responses for all four questions were collected on 7-point Likert scales from "strongly disagree" to "strongly agree" (midpoint = "neither disagree nor agree"). For perceived risk, we asked for participant's level of agreement with two statements, which were combined to create a scale (Cronbach's $\alpha=0.87$ ): (1) "Urine-derived fertilizer will be harmful to my health and the heath of my family" and (2) "Urine-derived fertilizer will be harmful to the environment." Similarly, for perceived benefits, we also asked for participant's level of agreement with two statements, which were combined to create a scale (Cronbach's $\alpha=0.88$ ): (1) “Using urine-derived fertilizer will result in foods that are healthier for people" and (2) "Using urine-derived fertilizer will be healthy for the environment overall."

With respect to individual characteristics that might moderate the effectiveness of different risk communication strategies, we focused on two main psychological covariates, apart from demographics. First, we assessed participants' level of food disgust sensitivity using the 8-item Food Disgust Scale (Cronbach's $\alpha=0.76$ ) developed and validated by Ammann and colleagues [49]. We did so because the idea of fertilizers derived from human urine may prompt food-related disgust, and we wanted to test the effect of these feelings on different risk communication strategies. Responses to these 8-items were collected on 6-point Likert scales ranging from $1=$ "Not disgusting at all" to $6=$ "Extremely disgusting"; the midpoint was not labeled. The final scale measured participants' value orientations, specifically as they related to the environment and sustainability. Here, participants responded to questions used for the biospherism subscale (four items that focused on one's consideration of environmental well-being; Cronbach's $\alpha=0.91$ ) of the value orientations battery developed by de Groot and Steg [50]. Lastly, we asked for demographic information from participants, including age, education level, and gender.

\subsection{Analysis}

We conducted analyses of variance with Tukey's post-hoc comparisons to detect differences between the control group and other communication strategies across the main study variables: usefulness of the communication strategy, perceptions of risk and benefit, and ratings of acceptability. To account 
for the prospect of Type II errors due to multiple comparisons, we applied a Bonferroni correction; thus, the $p$-value required for significance in the ANOVAs and post-hoc assessments was set at 0.01 . In addition, we conducted moderation analyses through hierarchical linear regression to assess how age and education impacted perceptions of usefulness differently if participants read text or watched videos. Moderation analyses for food disgust sensitivity and biospherism were likewise conducted and can be found in the Supplementary Information section.

We also performed a hierarchical linear regression to assess how the different risk communication strategies alongside demographic factors (age, gender, and education), value orientations, food disgust sensitivity, and perceptions of HUDF's risks, benefits, and naturalness explained ratings of acceptance of HUDF. This regression only explored acceptance of HUDF use on products intended for human consumption. The first step included the risk communication treatments and demographic characteristics, the second included value orientations and food disgust sensitivity, and the third added the risk, benefit and naturalness perceptions.

\section{Results}

\subsection{Comparisons of Communication Strategies}

When assessing the usefulness of the different risk communications about HUDF, an ANOVA detected a main effect of risk communication strategy $(\mathrm{F}(52,989)=30.06, p<0.0001)$; see Table 2 . All communication strategies were perceived to be at least positively useful (all means being above the midpoint, zero). However, post-hoc testing exposed significant differences between the control and all other communication strategies; the control had the least useful information. There were also statistical differences between all other strategies except between the short video and long video and the short text and long text. The short video was, on average, perceived to provide more useful information compared to both the short and long texts.

Table 2. ANOVAs and Tukey's post-hoc tests comparing communication strategies: short text (ST), long text (LT), short video (SV), long video (LV), and the control (C). The Bonferroni corrected $p$-value required for significance was set at 0.01 .

\begin{tabular}{|c|c|c|c|c|c|c|c|c|c|c|c|c|}
\hline & \multicolumn{2}{|l|}{$\mathrm{C}$} & \multicolumn{2}{|c|}{ ST } & \multicolumn{2}{|c|}{ LT } & \multicolumn{2}{|c|}{ SV } & \multicolumn{2}{|c|}{ LV } & \multirow{2}{*}{$F$} & \multirow{2}{*}{$p$} \\
\hline & $\bar{x}$ & SE & $\bar{x}$ & SE & $\bar{x}$ & SE & $\bar{x}$ & SE & $\bar{x}$ & SE & & \\
\hline Usefulness & $4.94^{1,2,3,4}$ & 0.06 & $5.35^{6,7}$ & 0.05 & $5.36^{8,9}$ & 0.06 & 5.67 & 0.05 & 5.61 & 0.05 & 30.06 & $<0.0001$ \\
\hline Perceived Risk & $3.48^{2,3,4}$ & 0.07 & 3.13 & 0.07 & $3.03^{8}$ & 0.07 & 2.84 & 0.07 & 2.90 & 0.07 & 22.46 & $<0.0001$ \\
\hline $\begin{array}{l}\text { Perceived Benefit } \\
\text { Acceptability }\end{array}$ & $4.24^{1,2,3,4}$ & 0.06 & $4.73^{6,7}$ & 0.06 & 4.87 & 0.07 & 5.11 & 0.06 & 5.05 & 0.06 & 37.15 & $<0.0001$ \\
\hline Non-Edible Use & $4.85^{1,2,3,4}$ & 0.06 & 5.19 & 0.06 & 5.22 & 0.06 & 5.46 & 0.06 & 5.41 & 0.05 & 16.90 & $<0.0001$ \\
\hline $\begin{array}{c}\text { Human } \\
\text { Consumption }\end{array}$ & $3.97^{1,2,3,4}$ & 0.08 & $4.39^{6,7}$ & 0.08 & 4.62 & 0.08 & 4.96 & 0.07 & 4.91 & 0.07 & 25.83 & $<0.0001$ \\
\hline
\end{tabular}

An ANOVA (Table 2) also revealed a significant main effect of risk communication strategy on risk perceptions $(\mathrm{F}(52,988)=22.46, p<0.0001)$. Post-hoc testing discovered significant differences between the control and the short video, long video, and long text, wherein HUDF was perceived riskier to participants who read the control. There were no statistical differences between the control and short text, nor among the other strategies. An ANOVA (Table 2) also found a significant main effect of risk communication strategy on benefit perceptions $(\mathrm{F}(52,988)=37.15, p<0.0001)$. Post-hoc testing revealed statistical differences between the control and all other communication strategies; those in the control group perceived HUDF, on average, to have lower environmental and human health benefits. Both video groups also received statistically higher benefit ratings than the short text group.

Considering the acceptance of HUDF, an ANOVA detected statistically significant main effects of both non-edible $(\mathrm{F}(52,988)=16.90, p<0.0001)$ and edible uses $(\mathrm{F}(52,989)=25.83, p<0.0001)$; see Table 2 . The use of HUDF on non-edible crops was preferred to the use of HUDF on crops intended for human consumption across all communication strategies. Post-hoc testing showed that for both end-uses 
of HUDF, the control prompted the lowest ratings of acceptance when compared to the alternative risk communication strategies. Reading the short text also yielded significantly prompted levels of acceptance than watching either of the videos.

\subsection{Moderating Variables: Strategy, Age, and Education Level}

When assessing the usefulness of the risk communication strategies, the text strategies were not distinct from each other, nor were the video strategies (Table 2). For this reason, both the short and long texts were combined into a single "text" variable, and the short and long videos were combined into a single "video" variable to understand differences between the two modes of communication strategies. Age was divided into five categories: 18-29, 30-39, 40-48, 49-59, and 60+ (per Table 1). A hierarchical regression was conducted to determine how age and risk communication strategy affected the usefulness of the information presented. The first step of the regression tested the hypothesis that usefulness is a function of both age and strategy. These variables were both statically significant $(p<0.05)$. Next, the interaction of strategy and age was included, which was significant $(p=0.042)$. With the interaction term included, there was a full moderation effect; neither communication strategy, nor age alone, was statistically significant. The interaction plot (Figure 1, pane A) shows an increased effect of video on usefulness regardless of age group, but especially so for older individuals (49 and above).

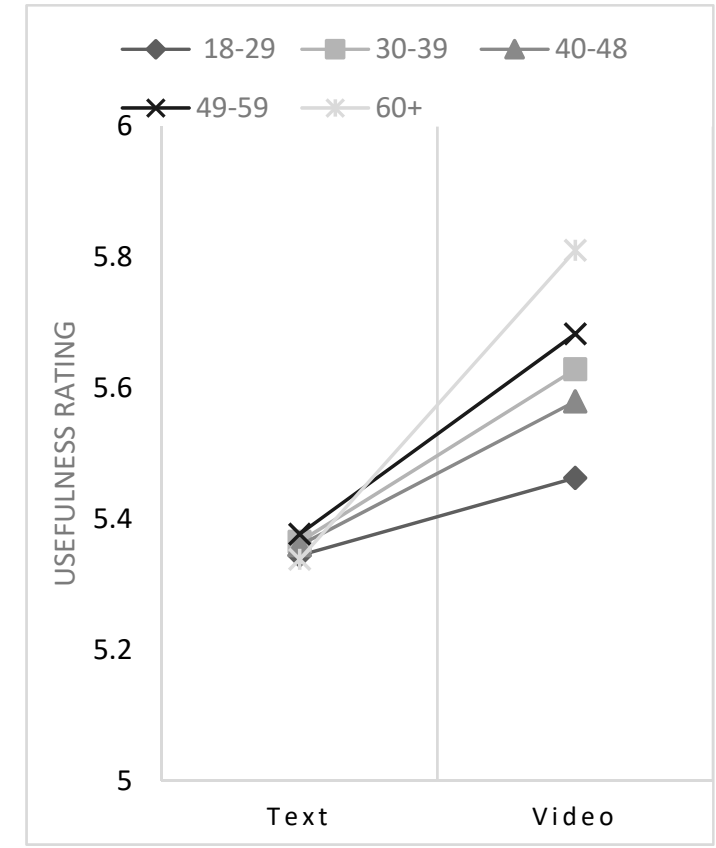

(a)

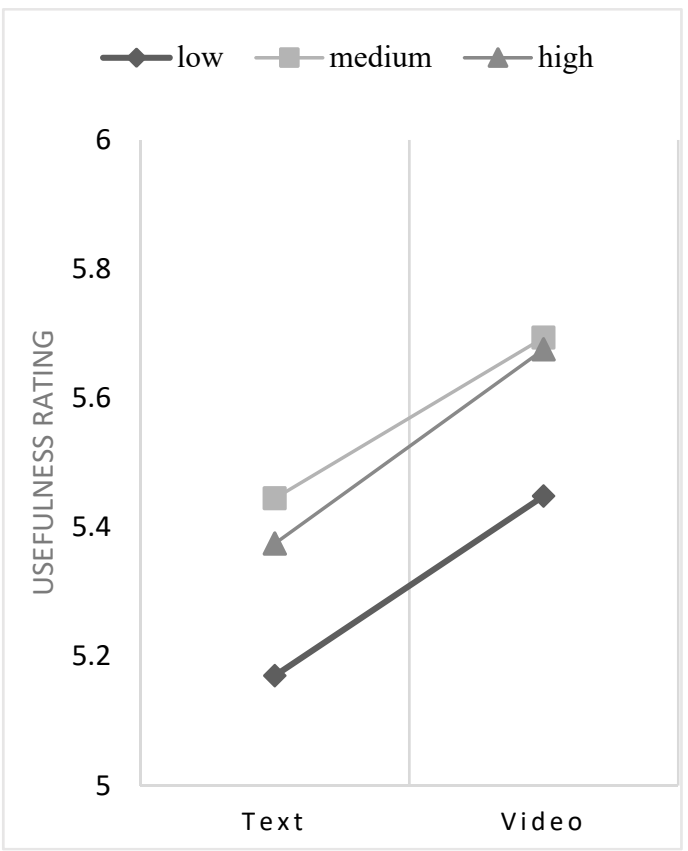

(b)

Figure 1. Interaction plots of usefulness of risk communication by (a) age and strategy, and (b) education level and strategy.

A second hierarchical linear regression was conducted to test the hypothesis that education and risk communication strategy would affect usefulness. Education was categorized as low (no high school, some high school, or graduated high school/GED), medium (some college or associate degree), or high (bachelor's degree or graduate/professional degree) per Table 1. The first step of the regression tested whether strategy and education were predictors of usefulness ( $p_{\text {strategy }}<0.000$; $p_{\text {loweducation }}=0.000$; pmediumeducation $=0.001 ;$ phigheducation $=0.002$ ). The second step involved an interaction between strategy and education level. There was no moderation effect; the relationships between strategy and medium education $(p=0.848)$ or high education $(p=0.873)$ were not statistically distinct from the reference group (low education, $p<0.000$ ). Once the interaction term was included, education levels 
remained significant $\left(\mathrm{p}_{\text {medium }}=0.010\right.$ and $\left.\mathrm{p}_{\text {high }}=0.042\right)$. The interaction plot (Figure 1, pane $\left.\mathrm{b}\right)$ indicates that video was generally preferred across all education levels, and those with low education found both communication strategies to be the least useful.

\subsection{Predictors of HUDF Acceptance}

The hierarchical linear regression assessing consumer acceptance of HUDF for use on products intended for human consumption (Table 3) was divided into three steps. The first step consisted of only demographic factors (age, gender, and education), as well as dummy variables for the other strategies (with the control group being the baseline). Gender was statistically significant with a small effect size $\left(\eta^{2}=0.02\right)$ in that men were, on average, more accepting of HUDF use than women. Note, a third category of gender was included in the survey instrument (non-binary, third gender). This category was not statistically distinct and had a small enough sample size that it was excluded from the analysis $(n=39)$. Education was also positively correlated and statistically significant with a small effect size $\left(\eta^{2}\right.$ $=0.003$ ). In this step, all four strategies were statistically distinct from the control group and had higher levels of acceptance. The second step included intuitive and ideological factors involved in people's understanding acceptance, namely, peoples' values and their sensitivity to disgusting food items. Gender continued to be significant with a small effect size $\left(\eta^{2}=0.001\right)$. Egoism and biospherism both had significant positive relationships with acceptance with small effect sizes $\left(\eta^{2}=0.004\right.$ and $\eta^{2}=0.02$, accordingly). Food disgust sensitivity was also a significant predictor of acceptance $\left(\eta^{2}=0.03\right)$ with an inverse relationship. Specifically, higher food disgust sensitivity led to lower levels of acceptance for HUDF use on crops intended for human consumption.

Table 3. Hierarchical linear regression results describing predictors of consumer acceptance of urine-derived fertilizers for human consumption.

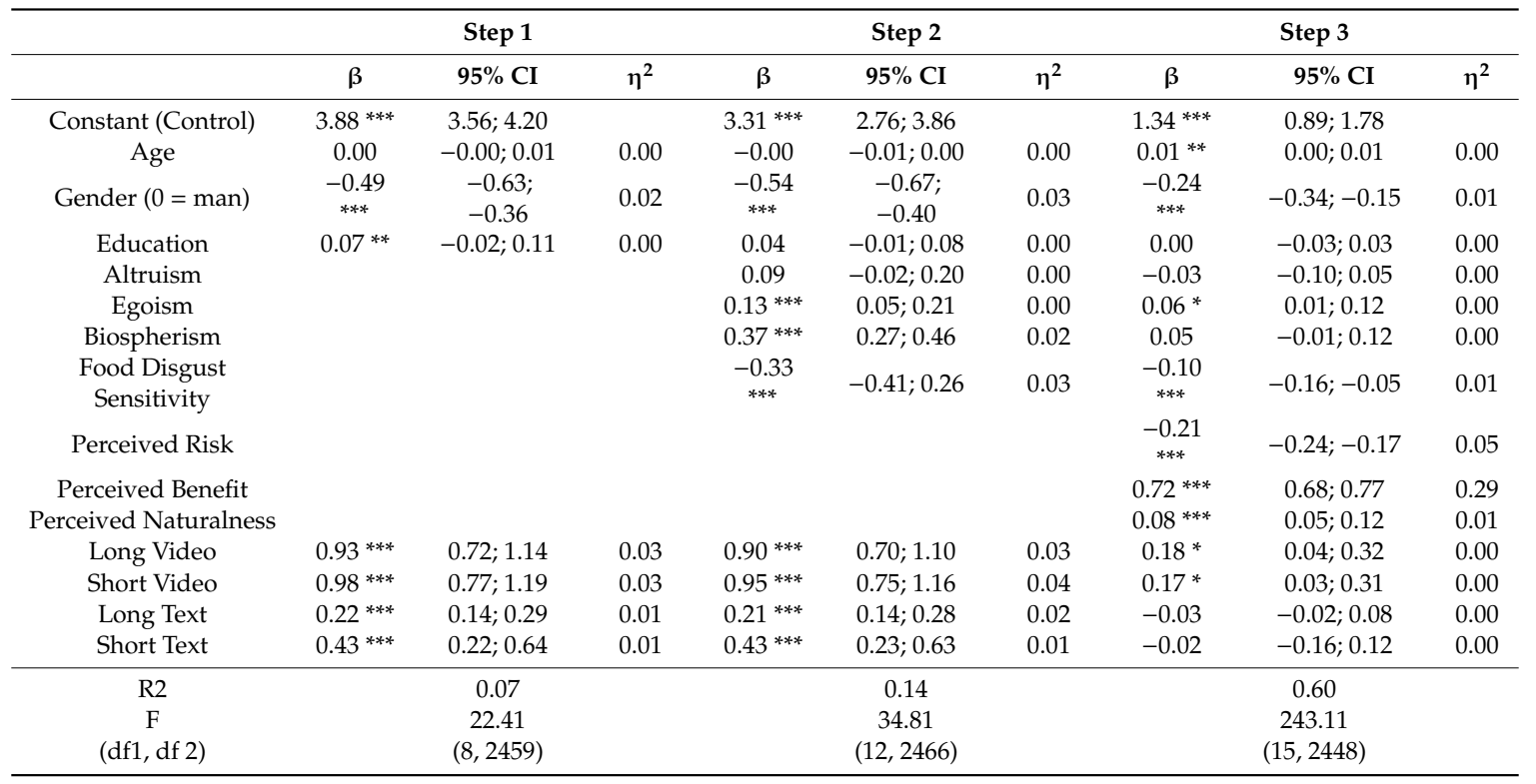

${ }^{*} p<0.05,{ }^{* *} p<0.01,{ }^{* * *} p<0.001$.

The third step went beyond solely instinctive (i.e., affect-laden) factors to incorporate cognitive components of acceptance, specifically perceptions of the risks, benefits, and naturalness of HUDF. We considered these variables as being more cognitive in nature because each communication strategy (except the control) had information regarding HUDF's risks, benefits, and naturalness. These three variables were all significant predictors of acceptance with varying effect sizes $\left(\eta^{2}\right.$ risk $=0.05$; $\eta^{2}{ }_{\text {benefit }}=0.29 ; \eta^{2}$ naturalness $=0.01$ ). As expected, risk and benefit perceptions were inversely related to each other, wherein benefit perceptions had a positive relationship with HUDF acceptance and risk perceptions were negatively correlated. Gender and egoism remained significant in the third step both 
with a small effect size $\left(\eta^{2}=0.01\right.$ and $\eta^{2}=0.002$, respectively). Additionally, age became statistically significant and positively correlated with acceptance, albeit with a small effect size $\left(\eta^{2}=0.004\right)$. In this last step of the regression, only the long video and the short video strategies were significantly distinct from the control.

\section{Discussion}

\subsection{The Usefulness of HUDF Risk Communication}

We observed statistically significant differences between the control group and all other risk communication strategies: short and long text, and short and long videos. Except for the mean risk perception score following exposure to the short text, all strategies outperformed the control across all of the variables we studied (Table 2). There were also some notable distinctions between the various risk communication strategies, namely, that the videos—and, specifically, the short video-outperformed both the short and long texts in participants' ratings of usefulness, perceived benefit of HUDF, and acceptance of HUDF use for use on plants and crops not intended for human consumption.

These findings build upon prior research; for example, other studies have shown that risk communication videos can outperform text containing the same information when it comes to improving people's understanding of novel topics and technologies, mitigating their perceptions of risk, and helping people to make better informed decisions [36,37,51]. In addition, videos have been shown in other research to both increase the level of engagement between people and the material being presented to them, as well as knowledge retention, when compared to people responding to the same information in written form [52].

These results also align with other research which found that videos, compared to other communication strategies, may be more effective to address consumer interests around how food is produced [53]. This may be even more pertinent to a novel food or food-related product, such as HUDF. Moreover, narrated videos, compared to texts, may improve consumer understanding, and help consumers make informed choices around food [53]. Not only are videos more effective at increasing knowledge, but participants also rated videos (compared to written materials) as more useful [54]. Videos may have led to a higher acceptability of HUDF than texts because of the multiple sensory experience videos have. Hearing and seeing material in tandem may be more convincing than just reading identical information. Other work suggests that videos may indeed be more helpful at building consumer trust toward food production [53].

In addition to studying how risk communication strategies effect perceptions of usefulness, we also sought to better understand how certain individual characteristics might also shape these perceptions. We found that the interaction between age risk communication strategy (text vs. video) fully moderated ratings of usefulness. In general, younger participants found both text and video strategies less useful than older participants. However, ratings of the usefulness of the risk communication videos were significantly higher than for text among older participants (Figure 1). These results confirmed our hypothesis that older individuals would find videos to be more useful than their younger counterparts, and they align with prior studies that suggest that age moderates the effects of usefulness when considering different risk communication strategies [55]. Older adults also seem to prefer succinct materials and they also especially find videos more helpful than text at explaining new information $[35,56]$. This may be because episodic memory retention is easier for older adults than other memory retention options, and it is easier to activate episodic memory through videos over text [57].

We did not find ratings of usefulness for the different risk communication strategies to be moderated by self-reported education level. We initially hypothesized that those with lower levels of education may perceive video to be more useful than text. This is particularly because videos have been shown to be easier to understand and increase information retention and knowledge, especially for populations with lower levels of education or literacy [58,59]. However, much of the literature on this 
topic is related to informing medical patients in clinical practices. In that domain, general education level may in fact be a main driver of usefulness of communication strategy. It may be the case that asking about domain-specific knowledge, such as familiarity with fertilization practices and farming, may have been a better indicator than general education levels.

\subsection{Risk Communication and HUDF Acceptance}

Our hierarchical regression results indicated that perceptions of both HUDF risk and, especially, benefit, were the strongest predictors of HUDF acceptance; this finding is in line with prior research $[19,60]$. However, there were other variables that also played an explanatory role in HUDF acceptance. We hypothesized based on other research that biospherism and egoism would both be related to acceptance $[50,60]$, as the former is emblematic of concern for the environment and the latter is emblematic of a deep care for personal well-being to which consumption is closely tied. In the third step of the regression, egoism was the only statistically significant value orientation. In hindsight, this relationship makes intuitive sense because consumers could continue to act their usual ways, but the ingredients of their purchases would change. This technological change, that would not require individuals to act differently, may be appealing to those with higher levels of egoism, as it aligns with values of self-interest [24].

We also hypothesized that people exhibiting higher levels of food disgust sensitivity would exhibit lower levels of HUDF acceptance, and that people who associated higher levels of perceived naturalness with HUDF would be more accepting. Both of these hypotheses were supported, albeit with small effect sizes $\left(\eta^{2}=0.01\right)$. Perceived naturalness was positively related to acceptance of HUDF, which supports a trend found in a recent systematic review of this variable and food technology acceptance [61]. Perceived naturalness can be understood through a product's origin, production, and final outcome [61]. It makes sense, then, that participants perceived HUDF as natural, since the ingredients, final product, and, in some cases, the production process as well, are all accomplished through "natural" means.

As a counterweight to perceived naturalness, we also included food disgust sensitivity as a covariate in our model. Our results on disgust sensitivity were in line with other research, where this variable regularly has an inverse relationship with food technology acceptance, and especially for products that can easily be linked to waste (e.g., human urine-derived fertilizer, in the case of this research) $[19,43,49]$. As we note in the introduction, while human urine may be natural, it may also trigger feelings of disgust as it relates to waste. It seems that HUDF was indeed disgust inducing, and this disgust negatively affected risk communication efforts and consumers' receptivity to it.

Age was a positive and statistically significant predictor of acceptance in our research. Some studies have shown that older individuals are more open-minded and accepting of new technologies [62], though findings linking age to acceptance have been mixed [63]. These findings, combined with the results of our moderation analyses, indicate that more work may be needed to address how different age groups may respond to risk communication strategies about novel technologies and, specifically, HUDF.

\section{Conclusions}

Human urine-derived fertilizer is not yet widely available for consumers or farmers to purchase. As a result of this, the present study focused on the usefulness of the communication strategy and general acceptance, rather than consumer behavior. However, if HUDF does come to the market, future studies should explore how different communication strategies that include a perceived efficacy message may change behavior over just perceived acceptance. Efficacy, in this context, refers to people's sense of being able to do something to either produce a positive outcome or prevent a negative one $[64,65]$. As one becomes more familiar with a product, it is likely that one's self-efficacy around it increases [66]. Messages, therefore, that frame a product as something that people are more familiar 
with, that they have encountered before, and provide ways to use it effectively, may have a higher likelihood of influencing both hypothetical acceptability and actual behavior change.

In the end, the results from this study may be of use to not only risk communication researchers, but also practitioners interested in developing educational materials for the general public on waste related technology and, specifically, HUDF. These results are promising, as they indicate general open mindedness to HUDF, especially when the information is communicated in a succinct and video-based format.

Moreover, our results illuminated the fact that the short and long videos were the most useful risk communication strategies, and that HUDF was most accepted by individuals who watched either video over those individuals who read either text. As there were no significant distinctions between the short and long videos, practitioners interested in using videos for communicating risks may be better served by focusing their resources and attention on shorter options.

Finally, as there are parallels between HUDF and other novel food technologies (e.g., cultured meat, insect protein, and food nanotechnology), this research may serve as a jumping off point for future studies focused on how risk communication strategies may affect consumer acceptance of them.

Supplementary Materials: The following are available online at http://www.mdpi.com/2071-1050/12/23/9973/s1, Video S1: Short Video, Video S2: Long Video, Figure S1: Short Text, Figure S2: Long Text, Table S1: Survey Instrument.

Author Contributions: Conceptualization, A.S.C. and J.Á.; methodology, A.S.C. and J.Á.; software, A.S.C., N.G.L., and J.Á.; validation, A.S.C. and J.Á.; formal analysis, A.S.C. and J.Á.; investigation A.S.C. and J.Á.; resources, A.S.C., N.G.L., and J.Á.; data curation, A.S.C. and J.Á.; writing-original draft preparation, A.S.C. and J.Á.; writing—review and editing, A.S.C., N.G.L, and J.Á.; visualization, A.S.C.; supervision, J.Á.; project administration, A.S.C., N.G.L, and J.Á.; funding acquisition, N.G.L. and J.Á. All authors have read and agreed to the published version of the manuscript.

Funding: This research was supported by the U.S. National Science Foundation under award numbers INFEWS 1639244 to the University of Michigan, and SES 1728807 to Decision Research and the University of Michigan. Any opinions, findings, and conclusions or recommendations expressed in this material are those of the authors and do not necessarily reflect the views of the National Science Foundation.

Acknowledgments: Thank you to Glen Daigger, Caitlin Drummond, Kim Nace, Lauren Lutzke, Abraham Noe-Hays, Audrey Pallmeyer, Linda Macpherson, and Tatiana Schreiber for their assistance with this research.

Conflicts of Interest: The authors declare no conflict of interest.

\section{References}

1. Frissel, M.J. Cycling of Mineral Nutrients in Agricultural Ecosystems; Elsevier: Amsterdam, The Netherlands, 1978.

2. Ashley, K.; Cordell, D.; Mavinic, D. A brief history of phosphorus: From the philosopher's stone to nutrient recovery and reuse. Chemosphere 2011, 84, 737-746. [CrossRef] [PubMed]

3. Stewart, W.M.; Dibb, D.W.; Johnston, A.E.; Smyth, T.J. The Contribution of Commercial Fertilizer Nutrients to Food Production. Agron. J. 2005, 97, 1-6. [CrossRef]

4. Smil, V. Phosphorus in the environment: Natural flows and human interferences. Annu. Rev. Energy Environ. 2000, 25, 53-88. [CrossRef]

5. Liang, S.; Qu, S.; Zhao, Q.; Zhang, X.; Daigger, G.T.; Newell, J.P.; Miller, S.A.; Johnson, J.X.; Love, N.G.; Zhang, L.; et al. Quantifying the Urban Food-Energy-Water Nexus: The Case of the Detroit Metropolitan Area. Environ. Sci. Technol. 2019, 53, 779-788. [CrossRef] [PubMed]

6. Kalmykova, Y.; Harder, R.; Borgestedt, H.; Svanäng, I. Pathways and Management of Phosphorus in Urban Areas. J. Ind. Ecol. 2012, 16, 928-939. [CrossRef]

7. Wu, J.; Franzén, D.; Malmström, M.E. Anthropogenic phosphorus flows under different scenarios for the city of Stockholm, Sweden. Sci. Total Environ. 2016, 542, 1094-1105. [CrossRef]

8. Wilsenach, J.; Van Loosdrecht, M. Impact of separate urine collection on wastewater treatment systems. Water Sci. Technol. 2003, 48, 103-110. [CrossRef]

9. Trimmer, J.T.; Margenot, A.J.; Cusick, R.D.; Guest, J.S. Aligning Product Chemistry and Soil Context for Agronomic Reuse of Human-Derived Resources. Environ. Sci. Technol. 2019, 53, 6501-6510. [CrossRef] 
10. Larsen, T.A.; Peters, I.; Alder, A.; Eggen, R.; Maurer, M.; Muncke, J. Re-engineering the toilet for sustainable wastewater management. Environ. Sci. Technol. 2001, 35, 192A-197A. [CrossRef]

11. Lind, B.-B.; Ban, Z.; Bydén, S. Volume reduction and concentration of nutrients in human urine. Ecol. Eng. 2001, 16, 561-566. [CrossRef]

12. Tarpeh, W.A.; Udert, K.M.; Nelson, K.L. Comparing Ion Exchange Adsorbents for Nitrogen Recovery from Source-Separated Urine. Environ. Sci. Technol. 2017, 51, 2373-2381. [CrossRef] [PubMed]

13. Christiaens, M.E.R.; Udert, K.M.; Arends, J.B.A.; Huysman, S.; Vanhaecke, L.; McAdam, E.; Rabaey, K. Membrane stripping enables effective electrochemical ammonia recovery from urine while retaining microorganisms and micropollutants. Water Res. 2019, 150, 349-357. [CrossRef] [PubMed]

14. Simha, P.; Senecal, J.; Nordin, A.; Lalander, C.; Vinnerås, B. Alkaline dehydration of anion-exchanged human urine: Volume reduction, nutrient recovery and process optimisation. Water Res. 2018, 142, 325-336. [CrossRef] [PubMed]

15. Simha, P.; Lalander, C.; Ramanathan, A.; Vijayalakshmi, C.; McConville, J.R.; Vinnerås, B.; Ganesapillai, M. What do consumers think about recycling human urine as fertiliser? Perceptions and attitudes of a university community in South India. Water Res. 2018, 143, 527-538. [CrossRef]

16. Lienert, J.; Larsen, T.A. High Acceptance of Urine Source Separation in Seven European Countries: A Review. Environ. Sci. Technol. 2010, 44, 556-566. [CrossRef]

17. Lienert, J.; Thiemann, K.; Kaufmann-Hayoz, R.; Larsen, T.A. Young users accept NoMix toilets-a questionnaire survey on urine source separating toilets in a college in Switzerland. Water Sci. Technol. 2006, 54, 403-412. [CrossRef]

18. Lamichhane, K.M.; Babcock, R.W., Jr. Survey of attitudes and perceptions of urine-diverting toilets and human waste recycling in Hawaii. Sci. Total Environ. 2013, 443, 749-756. [CrossRef]

19. Segrè Cohen, A.; Love, N.G.; Nace, K.K.; Árvai, J. Consumers' acceptance of agricultural fertilizers derived from diverted and recycled human urine. Environ. Sci. Technol. 2020, 54, 5297-5305. [CrossRef]

20. Kasperson, R.E.; Kasperson, J.X. The social amplification and attenuation of risk. Ann. Am. Acad. 1996, 545, 95-105. [CrossRef]

21. Kasperson, R.E.; Renn, O.; Slovic, P.; Brown, H.S.; Emel, J.; Goble, R.; Kasperson, J.X.; Ratick, S. The social amplification of risk: A conceptual framework. Risk Anal. 1987, 8, 177-187. [CrossRef]

22. Siegrist, M.; Cousin, M.-E.; Kastenholz, H.; Wiek, A. Public acceptance of nanotechnology foods and food packaging: The influence of affect and trust. Appetite 2007, 49, 459-466. [CrossRef] [PubMed]

23. L'Orange Seigo, S.; Arvai, J.; Dohle, S.; Siegrist, M. Predictors of risk and benefit perception of carbon capture and storage (CCS) in regions with different stages of deployment. Int. J. Greenh. Gas Control 2014, 25, $23-32$. [CrossRef]

24. Visschers, V.H.; Shi, J.; Siegrist, M.; Arvai, J. Beliefs and values explain international differences in perception of solar radiation management: Insights from a cross-country survey. Clim. Chang. 2017, 142, 531-544. [CrossRef]

25. Fielding, K.S.; Dolnicar, S.; Schultz, T. Public acceptance of recycled water. Int. J. Water Resour. Dev. 2019, 35, 551-586. [CrossRef]

26. Gamson, W.A.; Modigliani, A. Media discourse and public opinion on nuclear power. Am. J. Sociol. 1989, 95, 1-37. [CrossRef]

27. Flynn, J.; Kasperson, R.; Kunreuther, H.; Slovic, P. Time to rethink nuclear waste storage. Issues Sci. Technol. 1992, 8, 42-48.

28. Frewer, L. The public and effective risk communication. Toxicol. Lett. 2004, 149, 391-397. [CrossRef]

29. Árvai, J. The end of risk communication as we know it. J. Risk Res. 2014, 17, 1245-1249. [CrossRef]

30. Fischhoff, B. Risk Perception and Communication Unplugged: Twenty Years of Process. Risk Anal. 1995, 15, 137-145. [CrossRef]

31. Arvai, J.; Campbell-Arvai, V. Risk communication: Insights from the decision sciences. In Effective Risk Communication: Learning from the Past, Charting a Course for the Future; Arvai, J., Rivers, L., Eds.; Taylor \& Francis: London, UK, 2013; pp. 234-257.

32. Morgan, M.G.; Fischhoff, B.; Bostrom, A.; Atman, C.J. Risk Communication: A Mental Models Approach; Cambridge University Press: Cambridge, UK, 2002.

33. Rutsaert, P.; Regan, Á.; Pieniak, Z.; McConnon, Á.; Moss, A.; Wall, P.; Verbeke, W. The use of social media in food risk and benefit communication. Trends Food Sci. Technol. 2013, 30, 84-91. [CrossRef] 
34. Panagiotopoulos, P.; Barnett, J.; Bigdeli, A.Z.; Sams, S. Social media in emergency management: Twitter as a tool for communicating risks to the public. Technol. Forecast. Soc. Chang. 2016, 111, 86-96. [CrossRef]

35. John, D.R.; Cole, C.A. Age Differences in Information Processing: Understanding Deficits in Young and Elderly Consumers. J. Consum. Res. 1986, 13, 297-315. [CrossRef]

36. Krouse, H.J. Video modelling to educate patients. J. Adv. Nurs. 2001, 33, 748-757. [CrossRef] [PubMed]

37. Goldberg, M.H.; van der Linden, S.; Ballew, M.T.; Rosenthal, S.A.; Gustafson, A.; Leiserowitz, A. The Experience of Consensus: Video as an Effective Medium to Communicate Scientific Agreement on Climate Change. Sci. Commun. 2019, 41, 659-673. [CrossRef]

38. Visschers, V.H.M.; Meertens, R.M.; Passchier, W.F.; de Vries, N.K. Audiovisual risk communication unravelled: Effects on gut feelings and cognitive processes. J. Risk Res. 2008, 11, 207-221. [CrossRef]

39. Campbell-Arvai, V. Visits from the ghost of disturbance past: Information about past disturbance influences lay judgments of ecosystems. J. Environ. Manag. 2019, 232, 438-444. [CrossRef]

40. Siegrist, M. Factors influencing public acceptance of innovative food technologies and products. Trends Food Sci. Technol. 2008, 19, 603-608. [CrossRef]

41. Finucane, M.L.; Alhakami, A.; Slovic, P.; Johnson, S.M. The affect heuristic in judgments of risks and benefits. J. Behav. Decis. Mak. 2000, 13, 1-17. [CrossRef]

42. Wilson, R.S.; Arvai, J.L. When less is more: How affect influences preferences when comparing low and high-risk options. J. Risk Res. 2006, 9, 165-178. [CrossRef]

43. Curtis, V.; de Barra, M.; Aunger, R. Disgust as an adaptive system for disease avoidance behaviour. Philos. Trans. R. Soc. B: Biol. Sci. 2011, 366, 389-401. [CrossRef]

44. Rozin, P.; Fallon, A.E. A perspective on disgust. Psychol. Rev. 1987, 94, 23-41. [CrossRef] [PubMed]

45. Whitfield, S.C.; Rosa, E.A.; Dan, A.; Dietz, T. The Future of Nuclear Power: Value Orientations and Risk Perception. Risk Anal. 2009, 29, 425-437. [CrossRef] [PubMed]

46. Stern, P.C.; Dietz, T.; Abel, T.; Guagnano, G.A.; Kalof, L. A value-belief-norm theory of support for social movements: The case of environmentalism. Hum. Ecol. Rev. 1999, 6, 81-97.

47. Faul, F.; Erdfelder, E.; Buchner, A.; Lang, A.-G. Statistical power analyses using G*Power 3.1: Tests for correlation and regression analyses. Behav. Res. Methods 2009, 41, 1149-1160. [CrossRef] [PubMed]

48. United States Census Bureau 2017 American Community Survey 1-Year Estimates. Available online: https://factfinder.census.gov/faces/tableservices/jsf/pages/productview.xhtml?pid=ACS_17_1YR_ S0201\&prodType=table (accessed on 1 August 2020).

49. Ammann, J.; Hartmann, C.; Siegrist, M. Does food disgust sensitivity influence eating behaviour? Experimental validation of the Food Disgust Scale. Food Qual. Prefer. 2018, 68, 411-414. [CrossRef]

50. De Groot, J.I.; Steg, L. Value orientations to explain beliefs related to environmental significant behavior: How to measure egoistic, altruistic, and biospheric value orientations. Environ. Behav. 2008, 40, 330-354. [CrossRef]

51. Ludwig, S.; Schuelper, N.; Brown, J.; Anders, S.; Raupach, T. How can we teach medical students to choose wisely? A randomised controlled cross-over study of video- versus text-based case scenarios. BMC Med. 2018, 16. [CrossRef]

52. Yadav, A.; Phillips, M.M.; Lundeberg, M.A.; Koehler, M.J.; Hilden, K.; Dirkin, K.H. If a picture is worth a thousand words is video worth a million? Differences in affective and cognitive processing of video and text cases. J. Comput. High. Educ. 2011, 23, 15-37. [CrossRef]

53. Musto, M.; Cardinale, D.; Lucia, P.; Faraone, D. Influence of Different Information Presentation Formats on Consumer Acceptability: The Case of Goat Milk Presented as Obtained from Different Rearing Systems. J. Sens. Stud. 2015, 30, 85-97. [CrossRef]

54. Idriss, N.Z.; Alikhan, A.; Baba, K.; Amstrong, A.W. Online, Video-Based Patient Education Improves Melanoma Awareness: A Randomized Controlled Trial. Telemed. E-Health 2009, 15, 992-997. [CrossRef]

55. Tarhini, A.; Hone, K.; Liu, X. Measuring the Moderating Effect of Gender and Age on E-Learning Acceptance in England: A Structural Equation Modeling Approach for An Extended Technology Acceptance Model. J. Educ. Comput. Res. 2014, 51, 163-184. [CrossRef]

56. Thomas; Deary; Kaminski; Stockton; Zueew, D. Patients' preferences for video cassette recorded information: Effect of age, sex and ethnic group. Eur. J. Cancer Care 1999, 8, 83-86. [CrossRef] [PubMed]

57. Garg, V.; Camp, L.J.; Connelly, K.; Lorenzen-Huber, L. Risk Communication Design: Video vs. Text; Springer: Berlin/Heidelberg, Germany, 2012; pp. 279-298. 
58. Calderon, Y.; Haughey, M.; Bijur, P.E.; Leider, J.; Moreno-Walton, L.; Torres, S.; Gennis, P.; Bauman, L.J. An Educational HIV Pretest Counseling Video Program for Off-Hours Testing in the Emergency Department. Ann. Emerg. Med. 2006, 48, 27.e1. [CrossRef] [PubMed]

59. Murphy, P.W.; Chesson, A.L.; Walker, L.; Arnold, C.L.; Chesson, L.M. Comparing the effectiveness of video and written material for improving knowledge among sleep disorders clinic patients with limited literacy skills. South Med. J. 2000, 93, 297-304. [CrossRef] [PubMed]

60. Poortvliet, P.M.; Sanders, L.; Weijma, J.; De Vries, J.R. Acceptance of new sanitation: The role of end-users' pro-environmental personal norms and risk and benefit perceptions. Water Res. 2018, 131, 90-99. [CrossRef]

61. Román, S.; Sánchez-Siles, L.M.; Siegrist, M. The importance of food naturalness for consumers: Results of a systematic review. Trends Food Sci. Technol. 2017, 67, 44-57. [CrossRef]

62. Arning, K.; Ziefle, M. Different Perspectives on Technology Acceptance: The Role of Technology Type and Age; Springer: Berlin/Heidelberg, Germany, 2009; pp. 20-41.

63. Hauk, N.; Hüffmeier, J.; Krumm, S. Ready to be a Silver Surfer? A Meta-analysis on the Relationship Between Chronological Age and Technology Acceptance. Comput. Hum. Behav. 2018, 84, 304-319. [CrossRef]

64. Basil, M.; Witte, K. Health risk message design using the extended parallel process model. In Health communication Message Design: Theory and Practice; Cho, H., Ed.; Sage Publications: Thousand Oaks, CA, USA, 2012; pp. 41-58.

65. Bandura, A. Self-efficacy: Toward a unifying theory of behavioral change. Psychol. Rev. 1977, 84, $191-215$. [CrossRef]

66. Rogers, E. Diffusion of Innovations, 5th ed.; Free Press: New York, NY, USA, 2003.

Publisher's Note: MDPI stays neutral with regard to jurisdictional claims in published maps and institutional affiliations. 